\title{
TWIST SPINNING REVISITED
}

\author{
BY
}

\author{
DEBORAH L. GOLDSMITH AND LOUIS H. KAUFFMAN ${ }^{1}$
}

\begin{abstract}
This paper contains several applications of the following theorem: The 1-twist spin $L_{1}(k)$ of any knot $k \subset S^{n-1}$ is interchangeable with the standard unknotted $(n-2)$-sphere $K$ in $S^{n}$ by means of a homeomorphism of triples $h:{ }^{\prime}\left(S^{n}, K, L_{1}(k)\right) \rightarrow\left(S^{n}, L_{1}(k), K\right)$ which reverses the orientation of $S^{n}$, and preserves the orientations of $K$ and $L_{1}(k)$. One of these applications is Zeeman's Theorem about twist spun knots; another is a proof of a conjecture of R. H. Fox about certain manifolds which have the same fundamental group.

We also prove that the iterated twist spun knot $L_{a, b}(k) \subset S^{n+1}$ is fiber equivalent to one of $L_{0, g}(k)$ or $L_{8, g}(k)$ where $g=$ g.c.d. $(a, b)$.
\end{abstract}

Introduction. In this paper we return to E. C. Zeeman's original article Twisting spun knots [6]. Bringing to it our point of view, we are able to reprove his results in, we believe, an enlightening way, as well as to prove several new theorems which will be stated later on in this introduction. Throughout the paper we shall be working in the piecewise linear (PL) category. Thus homeomorphism means PL homeomorphism, and all manifolds are PL manifolds.

Our viewpoint, expressed in the lowest possible dimension, is the following (see [4]):

Let $S^{3}$ be the solid torus $D^{2} \times S^{1}$ with identifications $(x, \theta) \sim\left(x, \theta^{\prime}\right)$ on the boundary $x \in \partial D^{2}$, and denote the unknotted circle $\partial D^{2} \times \theta / \sim$ by $K^{1}$. This is a book structure on $S^{3}$ with binding $K^{1}$ and leaves $D^{2} \times \theta$. Suppose $K^{0} \subset D^{2}$ is a 0 -dimensional knot in the 2-dimensional disc, consisting of two points.

Define the $a$-twist spin of $K^{0}$ in $S^{3}$ to be the oriented knot (or link) in $S^{3}$ swept out by $K^{0}$ as it simultaneously twists through a rotation of $a \pi$ in the factor $D^{2}$, and spins through a rotation of $2 \pi$ in the factor $S^{1}$, as in Figure $A$. Observe that the $a$-twist spin of $K^{0}$ maps to the 1-twist spin of $K^{0}$ under the $a$-fold cyclic branched covering $p_{a}: S^{3} \rightarrow S^{3}$ of the 3-sphere branched along the unknotted circle $K^{1}$.

Received by the editors July 2, 1976.

AMS (MOS) subject classifications (1970). Primary 57C45; Secondary 55A25.

Key words and phrases. Manifold, knot, book structure, twist spinning.

'This research was partially supported by the National Science Foundation, NSF 76-07087 346 3254306. 


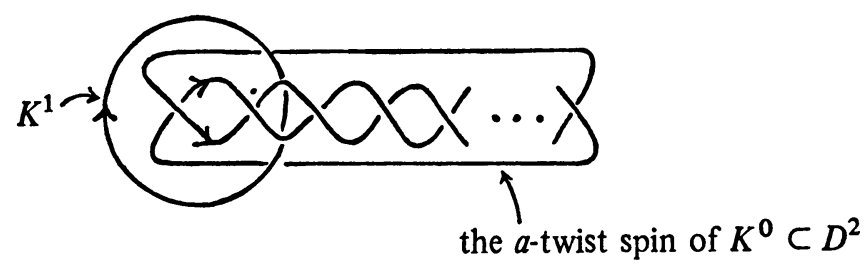

Figure A

The 1-twist spin of $K^{0}$ is obviously unknotted; in addition, it is interchangeable with the unknotted circle $K^{1}$ by a self-homeomorphism of $S^{3}$. (See Figure B.) Therefore, the $a$-twist spin of $K^{0}$ is actually equal to the inverse image $p_{a}^{-1}\left(K^{1}\right)$ of the circle $K^{1}$ under the $a$-fold cyclic branched covering $p_{a}: S^{3} \rightarrow S^{3}$ of the 3-sphere branched along the 1-twist spin of $K^{0}$.
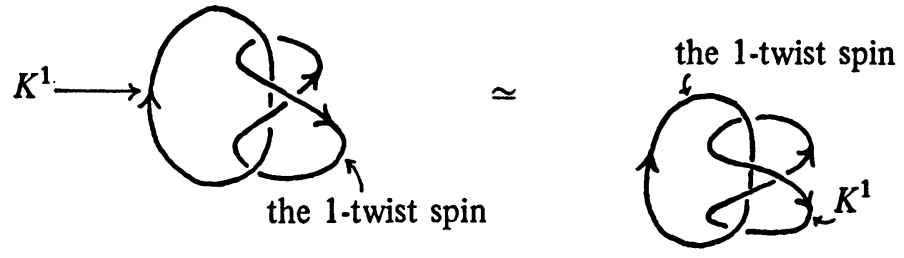

FIGURE B

Now, the 1-twist spin of $K^{0}$ meets each leaf $D^{2} \times \theta$ of the book structure on $S^{3}$ with binding $K^{1}$, in precisely two points. (The circle $K^{1}$ is said to be an axis for the 1-twist spin, or, equivalently, the 1-twist spin is a closed braid about $K^{1}$.) Consequently, the book structure on $S^{3}$ with binding $K^{1}$ and leaf $D^{2} \times \theta$ lifts to a book structure on the branched covering space $p_{a}: S^{3} \rightarrow S^{3}$ with binding $p_{a}^{-1}\left(K^{1}\right)$ and leaf $p_{a}^{-1}\left(D^{2} \times \theta\right)$. This gives a second book structure on $S^{3}$, whose leaves are the $a$-fold cyclic branched covering of the disc $D^{2} \times \theta, \theta \in S^{1}$, branched along two points, and whose binding is the $a$-twist spin of the 0 -dimensional knot $K^{0}$ (usually called a $(2, a)$ torus knot). This proves that the $(2, a)$ torus knot, $a$ odd, is a fibered knot of genus $(a-1) / 2$ (i.e., the complement of the $(2, a)$ torus knot is a surface bundle over the circle, whose fiber is the interior of a compact, connected, oriented surface with boundary in $S^{3}$, having $(a-1) / 2$ handles).

We are able to prove an analogous Interchange Theorem for the twist spun knots which Zeeman studied! The Interchange Theorem (1.10) states that "the 1-twist spin $L_{1}(k) \subset S^{n}$ of any knot $k \subset S^{n-1}$ is interchangeable with the standard, unknotted $(n-2)$-sphere $K$ in $S^{n}$ by means of a homeomorphism of triples $h:\left(S^{n}, K, L_{1}(k)\right) \rightarrow\left(S^{n}, L_{1}(k), K\right)$ which reverses the orientation of $S^{n}$, and preserves the orientations of $K$ and $L_{1}(k)$." Therefore the argument just presented, which we repeat in $\$ 2$, is a proof of Zeeman's theorem that is different from Zeeman's original proof. Zeeman's theorem (2.3) states that the $a$-twist spin $L_{a}(k)$ of a knot $k \subset S^{n-1}$ is a 
fibered knot, or (equivalently) the binding of a book structure on $S^{n}$, whose leaf is the $a$-fold cyclic branched cover of $S^{n-1}$ branched along $k \subset S^{n-1}$, with a disc removed.

In $\$ 3$ we use the Interchange Theorem to prove a conjecture of R. H. Fox, that the $a$-fold cyclic branched cover of $S^{n}$ branched along the $b$-twist spin $L_{b}(k)$ of the knot $k \subset S^{n-1}$ is homeomorphic to the $b$-fold cyclic branched cover of $S^{n}$ branched along the $a$-twist spin $L_{a}(k)$ of $k$.

$\$ 4$ proves that the iterated twist spun knots $L_{a, b}(k)$ and $L_{b, a}(k)$ in $S^{n+1}$ $\left(L_{x y}(k)\right.$ is the $y$-twist spin $L_{y}\left(L_{x}(k)\right) \subset S^{n+1}$ of the $x$-twist spin $L_{x}(k) \subset S^{n}$ of $k \subset S^{n-1}$ ) are fiber equivalent fibered knots, where the fibration of their complements over $S^{1}$ is given by Zeeman's theorem. The proof is similar to that of Fox's conjecture in $\$ 3$.

$\$ 5$ proves the special cases of the previous two theorems when either $a$ or $b$ is 0 .

In $\$ 6$ we state and prove a generalization of Zeeman's theorem for knotted submanifolds $k \subset S^{n-1}$, and describe the fiber of the fibration of the complement $S^{n}-L_{a}(k)$ over the circle.

In $\$ 7$ we prove the following two theorems:

THEOREM *. Let $k \subset S^{n-1}$ be a knot. Let $(a, b) \in \mathbf{Z} \times \mathbf{Z}-\{(0,0)\},(a, b)$ $=(g r, g s)$ where $g=$ g.c.d. $(a, b),(r, s) \in \mathbf{Z} \times \mathbf{Z}-\{(0,0)\}$ and g.c.d. $(r, s)=$ 1. Then the b-fold cyclic branched cover $M_{a, b}(k)$ of $S^{n}$ branched along the $a$-twist spin $L_{a}(k) \subset S^{n}$ of $k \subset S^{n-1}$ is homeomorphic to $M_{0,8}(k)$ if either $r$ or $s$ is even, and to $M_{g, g}(k)$ if both $r$ and $s$ are odd.

THEOREM **. Let $k \subset S^{n-1}$ be a knot. Let $(a, b) \in \mathbf{Z} \times \mathbf{Z}-\{(0,0)\}$, $(a, b)=(g r, g s)$ where $g=$ g.c.d. $(a, b),(r, s) \in \mathbf{Z} \times \mathbf{Z}-\{(0,0)\}$ and g.c.d. $(r, s)=1$. Then the iterated twist spin $L_{a, b}(k) \subset S^{n+1}$ of $k \subset S^{n-1}$ is fiber equivalent to $L_{0, g}(k)$ if either $r$ or $s$ is even, and to $L_{g, g}(k)$ if both $r$ and $s$ are odd, where the fiber of the fibration of their complements over $S^{1}$ is given by Zeeman's theorem.

We wish to thank Cameron Gordon for suggesting this problem to us. He has also proved results along these lines, which are described in $\$ 7$.

$\$ 8$ describes another application of the Interchange Theorem. Here we define the notion of an $a$-symmetric collection $C$ of knotted discs in $D^{n-1}$, $a \in \mathbf{Z}^{+}$, and of spinning such a collection to obtain a knotted ( $\left.n-2\right)$-sphere in $S^{n}$. We prove the theorem that every knotted $(n-2)$-sphere in $S^{n}$ produced in this way is equivalent (ambient isotopic) to the $a$-twist spin of a knot $k \subset S^{n-1}$ associated to $C$, and conversely.

\section{An interchange theorem.}

1.1 Some book structure preliminaries. A book structure on a closed, oriented manifold $M^{n}$ consists of a closed, oriented codimension-2 submanifold $k \subset$ 
$M^{n}$, called the binding of the book, and a fibration $M-k \rightarrow S^{1}$ of its complement over the circle. The closure in $M^{n}$ of each fiber is a compact, oriented, codimension-1 submanifold $F_{\theta}, \theta \in S^{1}$, called a closed leaf of the book, whose oriented boundary is $\partial F_{\theta}=k$; the interiors $\dot{F}_{\theta}$ are called open leaves of the book. The orientation on the bundle $M^{n}-k$, given by the product of the orientations on the fiber $\dot{F}_{\theta}$ and on the base $S^{1}$ (in that order), should agree with the orientation of $M^{n}$.

The manifold $M^{n}$ has a book structure with leaves $F_{\theta} \simeq F$ if and only if $M^{n}$ is the quotient space $M^{n}=F \times[0,1] /(x, 1) \sim(h(x), 0)$ for all $x \in F$, $(x, 0) \sim(x, t)$ for all $x \in \partial F, t \in[0,1]$, for some homeomorphism $h: F \rightarrow F$ which is the identity on the binding $\partial F$, and if $M^{n}$ is oriented by the product orientation on $F \times[0,1]$. The map $h$, called a monodromy for the book structure, is not unique; in fact, any isotopic deformation of $h$, relative to $\partial F$, produces the same book structure.

We will denote the book structure on $M^{n}$ by $F_{\theta}$. A presentation for the book structure $F_{\theta}$ is $\left(M^{n}, F, h\right)$, where we understand that $h: F \rightarrow F$ is a monodromy for $F$; thus the same book structure has many presentations.

Two book structures $F_{\theta}, F_{\theta}^{\prime}$ on $M$ are equivalent (denoted $F_{\theta} \equiv F_{\theta}^{\prime}$ ) if $F_{\theta} \cong F_{\theta}^{\prime} \cong F$, and there exists a homeomorphism $f: M \rightarrow M$ (which need not preserve orientation) preserving leaves.

Definition 1.2. A knot $k \subset S^{n}$ is an oriented, locally flat submanifold of the oriented $n$-sphere $S^{n}$, which is homeomorphic to $S^{n-2}$.

DEFINITION 1.3. A fibered knot $k \subset S^{n}$ is a knot which is the binding of some book structure on $S^{n}$.

Definition 1.4. Two knots $k, k^{\prime} \subset S^{n}$ are equivalent if there is a homeomorphism $f:\left(S^{n}, k\right) \rightarrow\left(S^{n}, k^{\prime}\right)$ (not necessarily orientation preserving on either $S^{n}$ or $k$ ).

Definition 1.5. Two fibered knots $k, k^{\prime} \subset S^{n}$ are fiber equivalent if they are equivalent by a homeomorphism $f$ which is also an equivalence of their associated book structures on $S^{n}$.

For the remainder of this section, and $\S \S 2,3,4,5$ and 7 it will be assumed that the binding of every book structure on $S^{n}$ is a knot.

LEMMA 1.6. Let $k \subset S^{n}$ be a closed, oriented, connected, locally flat, codimension-2 submanifold, and let $M$ denote the complement of an open regular neighborhood of $k$ in $S^{n}$. (Note that a closed regular neighborhood of $k$ in $S^{n}$ is homeomorphic to $k \times D^{2}$, whence $\partial M \cong k \times S^{1}$.) Then there is a map $p$ : $M \rightarrow S^{1}$ representing a generator of $H^{1}\left(S^{n}-k ; Z\right)$, such that $p \mid \partial M$ is the projection $k \times S^{1} \rightarrow S^{1}$ and $p$ is a fibration over all but finitely many $\theta \in S^{1}$. Hence, except for a finite number of $\theta^{\prime} s, F_{\theta}=p^{-1}(\theta)$ is a compact, codimension-1 submanifold of $M$, and $\partial F_{\theta}=k \times \theta$ is a longitude on the boundary $k \times S^{1}$ of the regular neighborhood of $k$ in $S^{n}$. 
Proof. This lemma is a standard result, and its proof will be omitted.

Notation. The circle is taken to be the quotient space $S^{1} \cong R^{1} / \theta \sim(\theta+$ $2 \pi$ ) for all $\theta \in \mathbf{R}^{1}$. By abuse of notation, we will sometimes write $\theta \in S^{1}$.

The $n$-sphere $S^{n}$ has a natural book structure whose leaves are $(n-1)$ balls $D_{\theta}^{n-1}$ and whose binding is an unknotted $(n-2)$-sphere $K^{n-2}$. It is given by the presentation $\left(S^{n}, D^{n-1}\right.$, id).

In the quotient space

$$
S^{n} \cong D^{n-1} \times S^{1} /(x, \theta) \sim\left(x, \theta^{\prime}\right) \text { for all } x \in \partial D^{n-1}, \theta, \theta^{\prime} \in S^{1},
$$

let $D_{\theta}^{n-1}$ denote $D^{n-1} \times \theta, \theta \in S^{1}$, and $i_{\theta}: D^{n-1} \rightarrow D_{\theta}^{n-1}$ be the inclusion $x \rightarrow(x, \theta)$; let $K^{n-2}$ be the $(n-2)$-sphere $\partial D^{n-1} \times S^{1} / \sim$ included in $S^{n} \simeq D^{n-1} \times S^{1} / \sim$.

Let $r_{\theta}: D^{n-1} \rightarrow D^{n-1}$ be rotation by $\theta \in S^{1}$ about the last coordinate axis, where $D^{n-1}=\left\{x \in \mathbf{R}^{n-1}:|x| \leqslant 1\right\}$.

Definimion 1.7. A fibered knot $L \subset S^{n}$ is an axis for a knot $L^{\prime} \subset S^{n}$ (equivalently $L^{\prime}$ is said to be spun about $L$ ) if there is a book structure $F_{\theta}$ on $S^{n}$ with binding $L$, and an orientation preserving homeomorphism $j: S^{n-2} \rightarrow$ $L^{\prime}$, which maps the natural book structure on $S^{n-2}$ into the book structure on $L^{\prime}$; that is, we require that:

(i) $L \cap L^{\prime}=j\left(K^{n-4}\right)$,

(ii) $j\left(D_{\theta}^{n-3}\right) \subset F_{\theta}$.

Notation. Let $k \subset S^{n}$ be a knot. Then $\left(D^{n}, \tilde{k}\right)$ will denote the oriented knotted ball pair obtained by removing from $S^{n}$ a tiny ball centered at a point of $k$. We say that $\tilde{k} \subset D^{n}$ is the knotted disc corresponding to the knotted sphere $k \subset S^{n}$.

REMARK 1.8. The knotted ball pair $\left(D^{n}, \tilde{k}\right)$ is independent of which point of $k$ we choose, and of which ball centered at that point is removed.

Definition 1.9 (Zeeman [6]). Let $k \subset S^{n-1}$ be a knot, and let $a \in Z$. The a-twist spin of $k$ in $S^{n}$ is a knot $L^{\prime} \subset S^{n}$ spun about the standard unknotted sphere $K^{n-2}$, which satisfies

$$
j\left(D_{\theta}^{n-3}\right)=i_{\theta} r_{a \theta}(\tilde{k}) .
$$

We will denote the $a$-twist spin of $k$ in $S^{n}$ by $K_{a}(k)$.

THEOREM 1.10 (INTERCHANGE THEOREM). The 1-twist spin $L_{1}(k)$ of any knot $k \subset S^{n-1}$ is interchangeable with the standard, unknotted sphere $K^{n-2}$ in $S^{n}$ by a homeomorphism of triples $h:\left(S^{n}, K, L_{1}(k)\right) \rightarrow\left(S^{n}, L_{1}(k), K\right)$ which reverses the orientation of $S^{n}$, and preserves the orientations of $K$ and $L_{1}(k)$.

Corollary 1.11 (Zeeman [6]). The 1-twist spin $L_{1}(k)$ of every knot $k \subset$ $S^{n-1}$ is unknotted in $S^{n}$.

Before proving the theorem, we will set up some notation: Let $\tilde{k} \times D^{2}$ be a trivial disc bundle neighborhood of the knotted disc $\tilde{k} \subset D^{n-1}$ associated to 
the knot $k \subset S^{n-1}$. Then $M=\operatorname{Cl}\left(D^{n-1}-\tilde{k} \times D^{2}\right)$ is the compact complement of $k$ in $S^{n-1}$. Its boundary, $\partial M$, is the union of two homeomorphic pieces, $A=\tilde{k} \times \partial D^{2}$ and $B=\mathrm{Cl}\left(\partial D^{n-1}-\tilde{k} \times D^{2}\right)$, which together form $S^{n-3} \times S^{1}$ (see Figure 1).

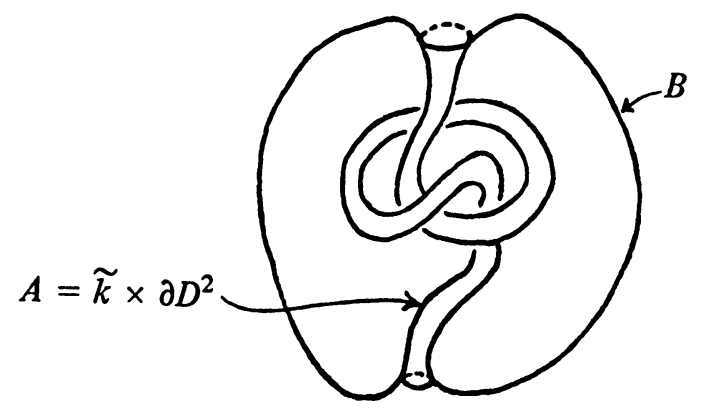

FIGURE 1

Let $B_{\theta}=D_{\theta}^{n-3} \cap B$ be the natural fibration induced on $B$ by the book structure $D_{\theta}^{n-3}$ on the $(n-2)$-sphere $\partial D^{n-1}$.

By Lemma 1.6 there is a mapping $p: M \rightarrow S^{1}$ such that $F_{\phi}=p^{-1}(\phi)$ is a surface except for finitely many $\phi \in S^{1}$, where it is not a manifold, and such that $p \mid \partial M \rightarrow S^{1}$ is a fibration whose fibers are longitudes of the knot $k$. We may assume that the longitude $p^{-1}(\phi) \cap \partial M$ consists of the disc $\tilde{k} \times \phi \subset \tilde{k}$ $\times \partial D^{2}$ in $A$, together with the disc $B_{\phi}$ in $B$.

Proof of Theorem 1.10. The triple $\left(S^{n}, L_{1}(k), K\right)$ is easily seen to be homeomorphic to the triple $\left(M \times S^{1}, A \times S^{1}, B \times S^{1}\right)$ after the identifications.

(i) $\tilde{k} \times \phi \times \theta \sim \tilde{k} \times \phi^{\prime} \times \theta$ for all $\tilde{k} \times \phi, \tilde{k} \times \phi^{\prime} \subset A, \theta \in S^{1}$,

(ii) $B_{\phi} \times \theta \sim B_{\phi^{\prime}} \times \theta^{\prime}$ whenever $\phi+\theta=\phi^{\prime}+\theta^{\prime}$.

We will define an orientation reversing homeomorphism $\tilde{h}: M \times S^{1} \rightarrow M \times$ $S^{1}$ which interchanges $A \times S^{1}$ and $B \times S^{1}$. The reader can check that $\tilde{h}$ is compatible with identifications (i) and (ii) on $M \times S^{1}$, and therefore induces the desired map $h: S^{n} \rightarrow S^{n}$.

Let $f: M \times S^{1} \rightarrow M \times S^{1}$ be defined by $F_{\phi} \times \theta \rightarrow F_{\phi} \times(-\theta-\phi), \theta, \phi$ $\in S^{1}$. (The map $f$ is the identity on the first factor. It reverses orientation because its restriction $f(x, \phi, \theta)=(x, \phi,-\theta-\phi)$ to the boundary $x \in \partial F_{0}$, $\theta, \phi \in S^{1}$, has negative determinant.)

Let $g=\tilde{g} \times$ id: $M \times S^{1} \rightarrow M \times S^{1}$, where $\tilde{g}: M \rightarrow M$ is an orientation preserving homeomorphism satisfying

(1) $F_{\phi}$ is invariant under $\tilde{g}$ for all $\phi \in S^{1}$,

(2) $\tilde{g}(A)=B$ and $\tilde{g}(B)=A$,

(3) $\tilde{g}$ is the identity outside of an open collar neighborhood of $\partial M$.

Finally, let $\tilde{h}=g \circ f$.

REMARK. Note that $\tilde{g}$ satisfies 
(4) $\tilde{g}(\tilde{k} \times \phi)=B_{\phi}$ and $\tilde{g}\left(B_{\phi}\right)=\tilde{k} \times \phi$ for all $\phi \in S^{1}$ as a consequence of (1)-(2). It is also the case that the restrictions $h \mid: L_{1}(k) \rightarrow K$ and $h \mid: K \rightarrow$ $L_{1}(k)$ are both orientation preserving.

REMARK. One can also obtain a homeomorphism of triples $h$ : $\left(S^{n}, K, L_{-1}(k)\right) \rightarrow\left(S^{n}, L_{1}(k), K\right)$ which is orientation preserving on $S^{n}$ and on the 1-twist spin $L_{-1}(k)$, and orientation reversing on $K$. Simply note that $L_{-1}(k)$ is homeomorphic to the triple $\left(M \times S^{1}, A \times S^{1}, B \times S^{1}\right)$ after the identifications (i) and

(ii') $B_{\phi} \times \theta \sim B_{\phi^{\prime}} \times \theta^{\prime}$ whenever $\theta-\phi=\theta^{\prime}-\phi^{\prime}$.

Then redefine the map $f: M \times S^{1} \rightarrow M \times S^{1}$ by $F_{\phi} \times \theta \rightarrow F_{\phi} \times(\theta-\phi)$, and let $\tilde{h}=g \circ f$, as before.

2. A new proof of Zeeman's theorem. In $\$ 2$ and $\$ 3$ we assume that the reader has some familiarity with branched covering spaces. Recall that if $X^{n}$ is a manifold and $Y^{n-2} \subset X^{n}$ is a subcomplex of dimension $(n-2)$, then given a representation $\sigma: \pi_{1}(X-Y) \rightarrow S(m)$ to the symmetric group on $m$ elements there is an associated branched covering $M \rightarrow X$, which is the unique completion (see [2]) of the covering space $\tilde{M} \rightarrow X-Y$ corresponding to $\sigma$. The set of points $Y$ over which $M \rightarrow X$ fails to be a covering space is called the singular, or branch, set.

Definition 2.1. Let $Y^{n-2} \subset X^{n}$ be an oriented, connected submanifold of the oriented manifold $X^{n}$, which is properly embedded in $X^{n}$ (i.e., $\partial Y=Y$ $\cap \partial X$ ). An oriented simple closed curve $m$ on the boundary of a regular neighborhood $N(Y) \subset X$ of $Y$ is called a positive meridian for $Y$ in $X$ if $m$ is the boundary of a fiber of the 2-disc bundle $N(Y) \rightarrow Y$, and the orientation of the fiber is such that it, together with the orientation of $Y$, induces an orientation on $N(Y)$ agreeing with that on $X$.

DefinItion 2.2. Let $Y^{n-2} \subset X^{n}$ be as in Definition 2.1, and suppose $H_{1}(X-Y ; Z) \cong Z$ is generated by the class $[m]$ of a positive meridian $m$ for $Y$ in $X$. The $a$-fold cyclic branched covering of $X$ branched along $Y$, $a \in \mathbf{Z}-\{0\}$, is the branched covering associated to the representation

$$
\pi_{1}(X-Y) \stackrel{i}{\rightarrow} H_{1}(X-Y ; \mathbf{Z}) \cong \mathbf{Z} \rightarrow \mathbf{Z} / a \mathbf{Z}
$$

where the last map is defined by mapping $[m] \rightarrow+1$. The branched covering space is given the orientation induced by $X$ if $a \in \mathbf{Z}^{+}$, and is given the opposite orientation if $a \in \mathbf{Z}^{-}$.

Notation. Let $k \subset S^{n-1}$ be a knot and $\tilde{k} \subset D^{n-1}$ be the knotted disc associated to $k$. Denote by $M_{a}(k) \rightarrow S^{n-1}$ the $a$-fold cyclic branched covering of $S^{n-1}$ branched along $k$. Denote by $M_{a}(\tilde{k}) \rightarrow D^{n-1}$ the $a$-fold cyclic branched covering of $D^{n-1}$ branched along $\tilde{k}$. Note that $M_{a}(\tilde{k})$ is obtained from $M_{a}(k)$ by removing an $(n-1)$-ball.

Let $p_{a}: S^{n} \rightarrow S^{n}$ be the $a$-fold cyclic branched covering of $S^{n}$ branched 
along $K^{n-2} \subset S^{n}$ induced by

$$
\begin{aligned}
\pi_{a}: D^{n-1} \times S^{1} & \rightarrow D^{n-1} \times S^{1}, \\
(x, \theta) & \rightarrow(x, a \theta) .
\end{aligned}
$$

Lemma 2.3. Let $k \subset S^{n-1}$ be a knot, $a \in \mathbf{Z}-\{0\}$, and let $p_{a}: S^{n} \rightarrow S^{n}$ be the a-fold cyclic branched covering of $S^{n}$ branched along the unknotted sphere $K^{n-2} \subset S^{n}$. Then the a-twist spin $L_{a}(k)$ of $k$ in $S^{n}$ is the inverse image $p_{a}^{-1}\left(L_{1}(k)\right) \subset S^{n}$ of the 1-twist spin of $k$ under the map $p_{a}$. The orientation of $L_{a}(k)$ agrees with the orientation of $p_{a}^{-1}\left(L_{1}(k)\right)$ if $a>0$, and is opposite to it if $a<0$.

Proof. It follows from the definition of $L_{a}(k)$ that $p_{a}\left(L_{a}(k)\right)=L_{1}(k)$, since

$$
\pi_{a}\left(r_{a \theta}(\tilde{k}) \times \theta\right)=r_{a \theta}(\tilde{k}) \times a \theta=r_{\phi}(\tilde{k}) \times \phi \text { for } \phi=a \theta .
$$

Checking orientations is left to the reader.

REMARK. Note that $M_{a}(k)$ is homeomorphic to $M_{-a}(k)$ by an orientation reversing map, and that the pairs $\left(S^{n}, L_{a}(k)\right)$ and $\left(S^{n}, L_{-a}(k)\right)$ are homeomorphic by a homeomorphism which is orientation reversing on both $S^{n}$ and $L_{a}(k)$.

COROllary 2.4. Let $k \subset S^{n-1}$ be a knot, $a \in \mathbf{Z}-\{0\}$, and let $\tilde{p}_{-a}$ : $S^{n} \rightarrow S^{n}$ be the $(-a)$-fold cyclic branched covering of $S^{n}$ branched along the unknotted sphere $L_{1}(k)$. Then the a-twist spin $L_{a}(k)$ of $k$ in $S^{n}$ is the inverse image $\left(\tilde{p}_{-a}\right)^{-\mathrm{i}}(K) \subset S^{n}$ of the standard, unknotted sphere $K^{n-2}$ under $\tilde{p}_{-a}$. The orientation of $L_{a}$ agrees with the orientation of $\tilde{p}_{-a}^{-1}(K)$ if $a>0$, and is opposite to it if $a<0$.

Proof. We have simply interchanged the roles of $L_{1}(k)$ and $K$ in Lemma 2.4, as an application of the Interchange Theorem. (Recall that the interchange map $h:\left(S^{n}, K, L_{1}(k)\right) \rightarrow\left(S^{n}, L_{1}(k), K\right)$ is orientation reversing on $S^{n}$, and therefore $p_{a}$ in Lemma 2.3 is replaced by $\tilde{p}_{-a}$ in Corollary 2.4.)

TheOREM 2.5 (ZeEMAN [6]). Let $k \subset S^{n-1}$ be any knot and let $a \in \mathbf{Z}-\{0\}$. Then the a-twist spin $L_{a}(k)$ of $k$ is a fibered knot in $S^{n}$; it is the binding of a book structure on $S^{n}$ with leaf $M_{-a}(\tilde{k})$.

Proof. We will use the characterization of $L_{a}(k)$ given by Corollary 2.4. The sphere $K^{n-2} \subset S^{n}$ is the binding of the book structure $D_{\theta}^{n-1}$ (see $\S 1$ ). Since $L_{1}(k)$ is spun about $K$, it meets each leaf $D_{\theta}^{n-1}$ in a homeomorphic copy of $\tilde{k}$, namely $i_{\theta} r_{\theta}(\tilde{k})$. Therefore the book structure $D_{\theta}^{n-1}$ lifts, under the branched covering map $\tilde{p}_{-a}: S^{n} \rightarrow S^{n}$, to a book structure on the branched covering space $S^{n}$ having binding $\left(\tilde{p}_{-a}\right)^{-1}(K) \cong L_{a}(k)$ and leaf $\left(\tilde{p}_{-a}\right)^{-1}\left(D^{n-1}\right) \simeq M_{-a}(\tilde{k})$. This is indicated in the following diagram: 


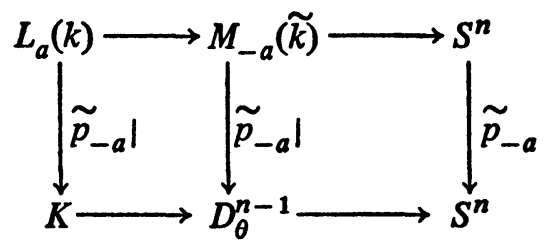

A close look also reveals the monodromy of the book structure, by which is meant an extension to the closed leaf $M_{-a}(\tilde{k})$ of a pasting map $\dot{M}_{-a}(\tilde{k}) \rightarrow$ $\dot{M}_{-a}(\tilde{k})$ for the fibration $S^{n}-L_{a}(k) \rightarrow S^{1}$ of the knot complement over the circle. A more complete discussion of this appears in $\$ 7$.

3. A conjecture of R. H. Fox. Let $k \subset S^{n-1}$ be a knot, $a, b \in \mathbf{Z}-\{0\}$; and define $\pi: M_{a, b}(k) \rightarrow S^{n}$ to be the $b$-fold cyclic branched covering of $S^{n}$ branched along the $a$-twist spin $L_{a}(k) \subset S^{n}$ of the knot $k$. R. H. Fox conjectured [3] that the spaces $M_{a, b}(k)$ and $M_{b, a}(k)$ are homeomorphic. We will now prove his conjecture.

In this section we assume $a, b \in \mathbf{Z}-\{0\}$. Theorem 3.1 is immediate from Lemmas 3.3 and 3.4, which follow.

THEOREM 3.1. Let $k \subset S^{n-1}$ be a knot. Then $M_{a, b}(k) \cong M_{b, a}(k)$; i.e., the $b$-fold cyclic branched covering of $S^{n}$ branched along the a-twist spin of $k \subset S^{n-1}$ is homeomorphic to the a-fold cyclic branched covering of $S^{n}$ branched along the b-twist spin of $k \subset S^{n-1}$.

We begin by defining the following branched covering of the sphere:

Definition 3.2. Let $k \subset S^{n-1}$ be a knot. Let $m_{1}, m_{2} \subset S^{n}-K \cup L_{1}(k)$ be positive meridians in $S^{n-1}$ for $K$ and $L_{1}(k)$, respectively, and identify

$$
H_{1}\left(S^{n}-K \cup L_{1}(k) ; \mathbf{Z}\right) \stackrel{\approx}{\rightarrow} \mathbf{Z} \oplus \mathbf{Z}
$$

by $\left[m_{1}\right] \rightarrow 1 \oplus 0$ and $\left[m_{2}\right] \rightarrow 0 \oplus 1$. Finally, let $\mathbf{Z} \stackrel{q_{m}}{\rightarrow} \mathbf{Z} / m \mathbf{Z}$ be the quotient map.

Define the branched covering space $X_{a, b}(k) \rightarrow S^{n}$ branched along the subcomplex $K \cup L_{1}(k) \subset S^{n}$ by the representation:

$$
\begin{aligned}
& \pi_{1}\left(S^{n}-K \cup L_{1}(k)\right) \stackrel{i}{\rightarrow} H_{1}\left(S^{n}-K \cup L_{1}(k) ; \mathbf{Z}\right) \stackrel{\Xi}{\rightarrow} \mathbf{Z} \oplus \mathbf{Z} \\
& \stackrel{q_{a} \oplus q_{b}}{\rightarrow}(\mathbf{Z} / a \mathbf{Z}) \oplus(\mathbf{Z} / b \mathbf{Z}) .
\end{aligned}
$$

LEMMA 3.3. $X_{a, b}(k) \cong M_{a, b}(k)$.

Proof. Since the branched covering $p_{a}: S^{n} \rightarrow S^{n}$ is determined by the representation:

$$
\pi_{1}\left(S^{n}-K\right) \stackrel{i_{*}}{\rightarrow} H_{1}\left(S^{n}-K ; Z\right) \stackrel{\approx}{\rightarrow} \mathrm{Z} \stackrel{q_{a}}{\rightarrow} \mathrm{Z} / a \mathrm{Z}
$$


and the branched covering $\pi: M_{a, b}(k) \rightarrow S^{n}$ is determined by the representation:

$$
\pi_{1}\left(S^{n}-L_{a}(k)\right) \stackrel{i_{*}}{\rightarrow} H_{1}\left(S^{n}-L_{a}(k) ; \mathbf{Z}\right) \stackrel{\sim}{\rightarrow} \mathbf{Z} \stackrel{q_{b}}{\rightarrow} \mathbf{Z} / b \mathbf{Z},
$$

and since $p_{a}\left(L_{a}(k)\right)=L_{1}(k)$ by Lemma 2.3, it follows that the composition $p_{a} \circ \pi: M_{a, b}(k) \rightarrow S^{n}$ is a branched covering with branch set $K \cup L_{1}(k) \subset$ $S^{n}$, determined by the representation (*). Hence $M_{a, b}(k) \cong X_{a, b}(k)$.

LEMMA 3.4. $X_{a, b}(k) \cong X_{b, a}(k)$.

Proof. It follows immediately from the Interchange Theorem that $X_{a, b}(k)$ $\cong X_{-b,-a}(k)$. Since the representation (*) has the same kernel if $a$ (or $b$ ) is replaced by $-a$ (resp., $-b$ ), we also have $X_{-b,-a}(k) \cong X_{b, a}(k)$.

REMARK. The homeomorphism $M_{a, b}(k) \rightarrow M_{b, a}(k)$ of Theorem 3.1 is orientation reversing.

4. Iterated twist spinning. Let $k \subset S^{n-1}$ be a knot, $a, b \in \mathbf{Z}-\{0\}$. We will let $L_{a, b}(k) \subset S^{n+1}$ denote the $b$-twist spin $L_{b}\left[L_{a}(k)\right] \subset S^{n+1}$ of the $a$-twist spin $L_{a}(k) \subset S^{n}$ of $k$. In this section we prove that $L_{a, b}(k)$ is fiber equivalent to $L_{b, a}(k)$. The proof is very similar to that of Fox's conjecture, and, in fact, Theorem 3.1 can be deduced as a corollary.

Notation. Let $U_{+}^{n-2}\left(U_{-}^{n-2}\right)$ be the upper (resp. lower) hemisphere of the boundary sphere $\partial D^{n-1}$. If $k \subset S^{n-1}$ is a knot, then the associated knotted arc $\tilde{k} \subset D^{n-1}$ is always placed so that $U_{+} \cap U_{-}$intersects $\partial \tilde{k}$ in its equator (if $n=4$, each hemisphere of $\partial D^{n-1}$ contains exactly one endpoint of $\tilde{k}$ ).

Represent $\left(S^{n+1}, L_{1,1}(k)\right)$ by the pair

$$
\left(D^{n-1} \times S^{1} \times S^{1}, \tilde{k} \times S^{1} \times S^{1}\right)
$$

with identifications

$$
\begin{aligned}
& (x, 0, \phi) \sim\left(r_{-\theta}(x), \theta, \phi\right) \quad \text { for all } x \in U_{-}, \theta, \phi \in S^{1}, \\
& (x, \theta, 0) \sim\left(r_{-\phi}(x), \theta, \phi\right) \quad \text { for all } x \in U_{+}, \theta, \phi \in S^{1} .
\end{aligned}
$$

Let $S_{+}^{n-1}$ be the oriented $(n-1)$-sphere $U_{+} \times S^{1} \times S^{1} / \sim$, and let $S_{-}^{n-1}$ be the oriented $(n-1)$-sphere $U_{-} \times S^{1} \times S^{1} / \sim$. The unknotted $(n-1)$ spheres $S_{+}, S_{-} \subset S^{n+1}$ intersect in general position in an $(n-3)$-sphere, $\left(U_{+} \cap U_{-}\right) \times S^{1} \times S^{1} / \sim$.

Let $P_{a}: S^{n+1} \rightarrow S^{n+1}$ be the $a$-fold cyclic branched cover of $S^{n+1}$ branched along $S_{-}$, induced by

$$
\begin{aligned}
p_{a}: D^{n-1} \times S^{1} \times S^{1} & \rightarrow D^{n-1} \times S^{1} \times S^{1}, \\
(x, \theta, \phi) & \rightarrow(x, a \theta, \phi) .
\end{aligned}
$$

Let $Q_{a}: S^{n+1} \rightarrow S^{n+1}$ be the $a$-fold cyclic branched cover of $S^{n+1}$ branched along $S_{+}$, induced by 


$$
\begin{aligned}
q_{a}: D^{n-1} \times S^{1} \times S^{1} & \rightarrow D^{n-1} \times S^{1} \times S^{1}, \\
(x, \theta, \phi) & \rightarrow(x, \theta, a \phi) .
\end{aligned}
$$

We would just as well let $\left(S^{n+1}, L_{1,1}(k)\right)$ be the pair (*) with identifications

$$
\begin{array}{ll}
(x, 0, \phi) \sim\left(r_{-\theta}(x), \theta, \phi\right) & \text { for all } x \in U_{+}, \theta, \phi \in S^{1}, \\
(x, \theta, 0) \sim\left(r_{-\phi}(x), \theta, \phi\right) & \text { for all } x \in U_{-}, \theta, \phi \in S^{1} .
\end{array}
$$

(This follows from Remark 1.8.) We will denote the pair $(*) /(* * *)$ by $\left(\tilde{S}^{n+1}, \tilde{L}_{1,1}(k)\right)$. Let $\tilde{S}_{ \pm}$be the $(n-1)$-sphere $U_{ \pm} \times S^{1} \times S^{1} /(* * *)$. Let $\tilde{P}_{a}$ : $\tilde{S}^{n+1} \rightarrow \tilde{S}^{n+1}$ be the $a$-fold cyclic branched cover of $\tilde{S}^{n+1}$ branched along $\tilde{S}_{+}$ which is induced by $p_{a}$, and let $\tilde{Q}_{a}: \tilde{S}^{n+1} \rightarrow \tilde{S}^{n+1}$ be the $a$-fold cyclic branched cover of $\tilde{S}^{n+1}$ branched along $\tilde{S}_{-}$which is induced by $q_{a}$.

LEMMA 4.1. $Q_{b} \circ P_{a}=P_{a} \circ Q_{b}: S^{n+1} \rightarrow S^{n+1}$ and $\tilde{Q}_{b} \circ \tilde{P}_{a}=\tilde{P}_{a} \circ \tilde{Q}_{b}: \tilde{S}^{n+1}$ $\rightarrow \tilde{S}^{n+1}$.

Proof. This follows because

$$
\begin{aligned}
q_{b} \circ p_{a}=p_{a} \circ q_{b}: D^{n-1} \times S^{1} \times S^{1} & \rightarrow D^{n-1} \times S^{1} \times S^{1}, \\
(x, \theta, \phi) & \rightarrow(x, a \theta, b \phi) .
\end{aligned}
$$

Definition 4.2. I: $\left(S^{n+1}, L_{1,1}(k)\right) \rightarrow\left(\tilde{S}^{n+1}, \tilde{L}_{1,1}(k)\right)$ is the orientation reversing homeomorphism induced by

$$
\text { i: } \begin{gathered}
D^{n-1} \times S^{1} \times S^{1} \rightarrow D^{n-1} \times S^{1} \times S^{1}, \\
(x, \theta, \phi) \rightarrow(x, \phi, \theta) .
\end{gathered}
$$

( $I$ reverses the orientation of both factors.)

LEMMA 4.3. The homeomorphism I: $\left(S^{n+1}, L_{1,1}(k)\right) \rightarrow\left(\tilde{S}^{n+1}, \tilde{L}_{1,1}(k)\right)$ preserves the book structures corresponding to the iterated twist spun knot $L_{1,1}(k)$ and $\tilde{L}_{1,1}(k)$, given by Zeeman's theorem.

Proof. Let $p: D^{n-1}-\tilde{k} \rightarrow S^{1}$ be the singular fibration described in Lemma 1.4, with $F_{\phi}=p^{-1}(\phi)$. The leaves $\mathscr{F}_{\theta}$ of the book structure on $S^{n}$ with binding $L_{1}(k)$ given by Zeeman's theorem are $\mathscr{F}_{\theta}=\cup_{\phi \in S^{1}} F_{\phi} \times(\theta-\phi)$ (see, for example, the proof of Theorem 1.10, or Theorem 6.4). The leaves $\mathcal{G}_{\alpha}$ of the book structure on $S^{n+1}$ with binding $L_{1,1}(k)$ are therefore

$$
\begin{aligned}
\mathcal{G}_{\alpha} & =\bigcup_{\theta \in S^{\prime}} \mathscr{F}_{\theta} \times(\alpha-\theta) \\
& =\bigcup_{\phi, \theta \in S^{\prime}} F_{\phi} \times(\theta-\phi) \times(\alpha-\theta)=\bigcup_{\theta, \phi \in S^{\prime}} F_{\alpha-\theta-\phi} \times \theta \times \phi .
\end{aligned}
$$

Now it is clear that $I\left(\mathcal{G}_{\alpha}\right)=\mathcal{G}_{\alpha}$ for all $\alpha \in S^{1}$.

THEOREM 4.4. Let $k \subset S^{n-1}$ be a knot; $a, b \in \mathbf{Z}-\{0\}$. Then the iterated twist spun knots $L_{a, b}(k)$ and $L_{b, a}(k)$ in $S^{n+1}$ are fiber equivalent fibered knots, 
where the fibration of their complements over $S^{1}$ is given by Zeeman's theorem.

Proof. By two applications of Lemma 2.3, we have that the iterated twist spun knot $L_{x y}(k) \subset S^{n+1}$ is the inverse image $\left[Q_{y} \circ P_{x}\right]^{-1}\left(L_{1,1}(k)\right)$ of the trivial knot $L_{1,1}(k) \subset S^{n+1}$ under the branched covering map $Q_{y} \circ P_{x}: S^{n+1}$ $\rightarrow S^{n+1}$; likewise, we also have that $L_{x y}(k)$ is the inverse image $\left[\tilde{Q}_{y} \circ \tilde{P}_{x}\right]^{-1}\left(\tilde{L}_{1,1}(k)\right)$ of $\tilde{L}_{1,1}(k)$ under the branched covering $\tilde{Q}_{y} \circ \tilde{P}_{x}: \tilde{S}^{n+1} \rightarrow$ $\tilde{S}^{n+1}$.

Now observe that the following diagram is commutative:

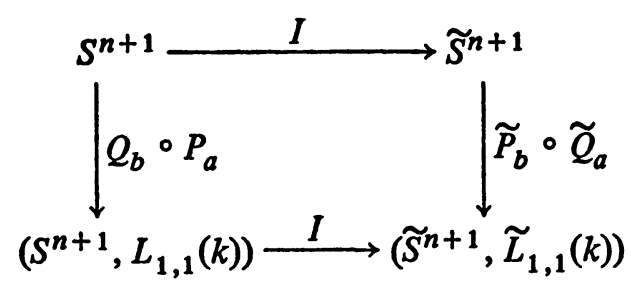

Finally, since $\tilde{P}_{b} \circ \tilde{Q}_{a}=\tilde{Q}_{a} \circ \tilde{P}_{b}: \tilde{S}^{n+1} \rightarrow \tilde{S}^{n+1}$, we have that $\tilde{L}_{b, a}(k)=$ $\left[\tilde{Q}_{a} \circ \tilde{P}_{b}\right]^{-1}\left(\tilde{L}_{1,1}(k)\right)=\left[\tilde{P}_{b} \circ \tilde{Q}_{a}\right]^{-1}\left(\tilde{L}_{1,1}(k)\right)$; hence,

$$
\begin{aligned}
\left(S^{n+1}, L_{a, b}(k)\right) & =\left(S^{n+1},\left[Q_{b} \circ P_{a}\right]^{-1}\left(L_{1,1}(k)\right)\right) \\
& \geqq\left(\tilde{S}^{n+1},\left[\tilde{P}_{b} \circ \tilde{Q}_{a}\right]^{-1}\left(\tilde{L}_{1,1}(k)\right)\right)=\left(\tilde{S}^{n+1}, \tilde{L}_{b, a}(k)\right) .
\end{aligned}
$$

Furthermore, since $I:\left(S^{n+1}, L_{1,1}(k)\right) \rightarrow\left(\tilde{S}^{n+1}, \tilde{L}_{1,1}(k)\right)$ is an equivalence of book structures, then $I:\left(S^{n+1}, L_{a, b}(k)\right) \rightarrow\left(\tilde{S}^{n+1}, \tilde{L}_{b, a}(k)\right)$ is also an equivalence of the book structures induced on the branched covering spaces, which is precisely that given by Zeeman's theorem.

REMARK. Fox's conjecture is actually a corollary of Theorem 4.4. This is because the fiber equivalence $I:\left(S^{n+1}, L_{a, b}(k)\right) \rightarrow\left(\tilde{S}^{n+1}, \tilde{L}_{b, a}(k)\right)$ carries the leaf $M_{-a,-b}(\tilde{k})$ homeomorphically to the leaf $M_{-b,-a}(\tilde{k})$ (in an orientation reversing manner). It is interesting to note that the Interchange Theorem is not used in the proof of Theorem 4.4.

5. The special case $a$ or $b$ is zero. We will now extend the theorems of $\S 2$, $\S 3$, and $\$ 4$ to include the cases where $a$ or $b$ is zero.

Definition 5.1. Let $S^{n}$ be the space with identifications $D^{n-1} \times S^{1} /(x, \partial)$ $\sim\left(x, \theta^{\prime}\right)$ for all $x \in \partial D^{n-1}, \theta, \theta^{\prime} \in S^{1}$. Then define $p_{0}: S^{n} \rightarrow D^{n-1}$ to be induced by projection $D^{n-1} \times S^{1} \rightarrow D^{n-1}$. (Note. If $S^{n}$ is represented as the space with identifications $D^{n-1} \times S^{1} /(x, 0) \sim\left(r_{a \theta}(x), \theta\right)$ for all $x \in \partial D^{n-1}$, $\theta \in S^{1}$, then $p_{0}: S^{n} \rightarrow D^{n-1}$ is induced by

$$
\begin{aligned}
D^{n-1} \times S^{1} & \rightarrow D^{n-1}, \\
(x, \theta) & \left.\rightarrow r_{-a \theta}(x) .\right)
\end{aligned}
$$


Definition 5.2. Let $S^{n+1}$ be the space (*) $D^{n-1} \times S^{1} \times S^{1}$ with identifications

$$
\begin{array}{ll}
(x, \theta, \phi) \sim\left(x, \theta^{\prime}, \phi\right) & \text { for all } x \in U_{-}, \theta, \theta^{\prime}, \phi \in S^{1}, \\
(x, \theta, \phi) \sim\left(x, \theta, \phi^{\prime}\right) & \text { for all } x \in U_{+}, \theta, \phi, \phi^{\prime} \in S^{\prime} .
\end{array}
$$

Then define $P_{0}: S^{n+1} \rightarrow S^{n+1}$ to be induced by

$$
\begin{aligned}
D^{n-1} \times S^{1} \times S^{1} & \rightarrow D^{n-1} \times S^{1} \times S^{1}, \\
(x, \theta, \phi) & \rightarrow(x, 0, \phi),
\end{aligned}
$$

and define $Q_{0}: S^{n+1} \rightarrow S^{n+1}$ to be induced by

$$
\begin{aligned}
D^{n-1} \times S^{1} \times S^{1} & \rightarrow D^{n-1} \times S^{1} \times S^{1}, \\
(x, \theta, \phi) & \rightarrow(x, \theta, 0) .
\end{aligned}
$$

Let $\tilde{S}^{n+1}$ be the space (*) with identifications

$$
\begin{array}{ll}
(x, \theta, \phi) \sim\left(x, \theta^{\prime}, \phi\right) & \text { for all } x \in U_{+}, \theta, \theta^{\prime}, \phi \in S^{1}, \\
(x, \theta, \phi) \sim\left(x, \theta, \phi^{\prime}\right) & \text { for all } x \in U_{-}, \theta, \phi, \phi^{\prime} \in S^{1} .
\end{array}
$$

Then define $\tilde{P}_{0}: \tilde{S}^{n+1} \rightarrow \tilde{S}^{n+1}$ to be induced by (1) and define $\tilde{Q}_{0}: \tilde{S}^{n+1} \rightarrow$ $\tilde{S}^{n+1}$ to be induced by (2).

(Note. If $S^{n+1}$ is represented as the space (*) with identifications

$$
\begin{array}{ll}
(x, 0, \phi) \sim\left(r_{a \theta}(x), \theta, \phi\right) & \text { for all } x \in U_{-}, \theta, \phi \in S^{1}, \\
(x, \theta, 0) \sim\left(r_{b} \phi(x), \theta, \phi\right) & \text { for all } x \in U_{+}, \theta, \phi \in S^{1},
\end{array}
$$

then $P_{0}: S^{n+1} \rightarrow S^{n+1}$ is induced by

$$
\begin{aligned}
D^{n-1} \times S^{1} \times S^{1} & \rightarrow D^{n-1} \times S^{1} \times S^{1}, \\
(x, \theta, \phi) & \rightarrow\left(r_{-a \theta}(x), 0, \phi\right)
\end{aligned}
$$

and $Q_{0}: S^{n+1} \rightarrow S^{n+1}$ is induced by

$$
\begin{aligned}
D^{n-1} \times S^{1} \times S^{1} & \rightarrow D^{n-1} \times S^{1} \times S^{1}, \\
(x, \theta, \phi) & \rightarrow\left(r_{-b \phi}(x), \theta, 0\right) .
\end{aligned}
$$

Similarly, if $\tilde{S}^{n+1}$ is represented as the space (*) with identifications

$(* * *)^{\prime}$

$$
\begin{array}{ll}
(x, 0, \phi) \sim\left(r_{a \theta}(x), \theta, \phi\right) & \text { for all } x \in U_{+}, \theta, \phi \in S^{1}, \\
(x, \theta, 0) \sim\left(r_{b \phi}(x), \theta, \phi\right) & \text { for all } x \in U_{-}, \theta, \phi \in S^{1},
\end{array}
$$

then $\tilde{P}_{0}: \tilde{S}^{n+1} \rightarrow \tilde{S}^{n+1}$ is induced by (1)' and $\tilde{Q}_{0}: \tilde{S}^{n+1} \rightarrow \tilde{S}^{n+1}$ is induced by (2)'. In $\S 4, S^{n+1} \cong(*) /(* *)^{\prime}$ and $\tilde{S}^{n+1} \cong(*) /(* * *)^{\prime}$ where $a=b=-1$.)

Definition 5.3. Let $K \subset S^{n}$ be a fibered knot, which is the binding of a book structure on $S^{n}$ with leaf $F$. Denote by $M_{0}(K)$ the underlying manifold of the book structure $\left(M_{0}(K), F, \mathrm{id}_{F}\right)$. Let $M_{0}(\tilde{K})$ be $M_{0}(K)$ minus an open ball centered at a point of $K=\partial F \subset M_{0}(K)$.

Definition 5.4. Let $k \subset S^{n-1}$ be a knot. In Definition 5.3, let $K$ be the 
fibered knot $L_{g}(k) \subset S^{n}, g \in \mathbf{Z}-\{0\}$, which is the binding of a book structure on $S^{n}$ with leaf $M_{g}(\tilde{k})$. Denote by $M_{g, 0}(k)$ the manifold $M_{0}\left(L_{g}(k)\right)$; denote by $M_{g, 0}(\tilde{k})$ the manifold $M_{0}\left(L_{g}(k)\right)$.

The following theorem is well known (see, for example, On higher-dimensional fibered knots by Andrews and Sumners, Trans. Amer. Math. Soc. 153 (1971), 415-426). The short proof which follows is our own:

THEOREM 5.5 (ZEEMAN'S THEOREM). Let $k \subset S^{n-1}$ be a fibered knot, which is the binding of a book structure on $S^{n-1}$ with leaf $F$. Then the 0-twist spin $L_{0}(k) \subset S^{n}$ is a fibered knot; it is the binding of a book structure on $S^{n}$ with leaf $M_{0}(\tilde{k})$.

Proof. The knot $L_{0}(k)$ is the inverse image $p_{0}^{-1}(\tilde{k})$ of the knotted disc $\tilde{k} \subset D^{n-1}$ under the mapping $p_{0}: S^{n} \rightarrow D^{n-1}$. The book structure on $S^{n-1}$ with binding $k$, when restricted to $\left(D^{n-1}, \tilde{k}\right)$, lifts to one on $S^{n}$ with binding $L_{0}(k)$ and leaf $p_{0}^{-1}(F) \cong M_{0}(\tilde{k})$.

CoRollary 5.6. Let $k \subset S^{n-1}$ be a knot, $g \in \mathbf{Z}-\{0\}$. Then the iterated twist spun knot $L_{g, 0}(k) \subset S^{n+1}$ is a fibered knot; it is the binding of a book structure on $S^{n+1}$ with leaf $M_{-g, 0}(\tilde{k})$.

Proof. Apply Theorem 5.5 to the fibered knot $L_{g}(k) \subset S^{n}$, which is the binding of a book structure on $S^{n}$ with leaf $M_{-g}(\tilde{k})$.

THEOREM 5.7. Let $k \subset S^{n-1}$ be a knot, $g \in \mathbf{Z}-\{0\}$. The iterated twist spun knots $L_{0,8}(k)$ and $L_{g, 0}(k)$ in $S^{n+1}$ are fiber equivalent fibered knots, where the fibration of their complement over $S^{1}$ is given by Zeeman's theorem.

PROof. The proof is identical to that of Theorem 4.4.

Corollary 5.8 (Fox's CONJECTURE). Let $k \subset S^{n-1}$ be a knot, $g \in \mathbf{Z}-$ $\{0\}$. Then $M_{0,8}(k) \cong M_{g, 0}(k)$.

PRoof. See the remark at the end of $\S 4$.

6. A generalization of Zeeman's theorem. Now let us suppose that $k \subset S^{n-1}$ is not a sphere. In this section we will employ the notation developed in $\$ 1$, reinterpreting $k$ as in 6.1:

DEFINITION 6.1. A knotted submanifold $k \subset S^{n-1}$ is an oriented, connected, codimension-2, locally flat submanifold of $S^{n-1}$.

Definition 6.2. A fibered knotted submanifold $k \subset S^{n-1}$ is a knotted submanifold of $S^{n-1}$ which is the binding of some book structure on $S^{n-1}$.

Definition 6.3. If $k \subset S^{n-1}$ is a knotted submanifold and $a \in \mathbf{Z}$, the $a$-twist spin $L_{a}(k) \subset S^{n}$ of $k$ is a knotted submanifold of $S^{n}$ which satisfies

$$
\left[L_{a}(k)\right] \cap D_{\theta}^{n-1}=i_{\theta} r_{a \theta}(\tilde{k}) \text {. }
$$

(As before, the pair $\left(D^{n-1}, \tilde{k}\right)$ is obtained from $\left(S^{n-1}, k\right)$ by removing a tiny ball centered at a point of $k$.) 
Notation. Recall the definition of $M$ from 1.10. Let $N$ be obtained from $M$ by making the identifications $B_{\phi} \sim B_{\phi^{\prime}}$ for all $\phi, \phi^{\prime} \in S^{1}$; thus $N$ is the compact complement in $S^{n-1}$ of a regular neighborhood of the knotted submanifold with boundary, $\tilde{k} \subset D^{n-1} \subset S^{n-1}$. Denote by $N_{a}(\tilde{k}) \rightarrow N$ the $a$-fold cyclic branched covering of $N$ branched along the complementary disc $\mathrm{Cl}(k-\tilde{k}) \subset N$. (See Figure 2.)

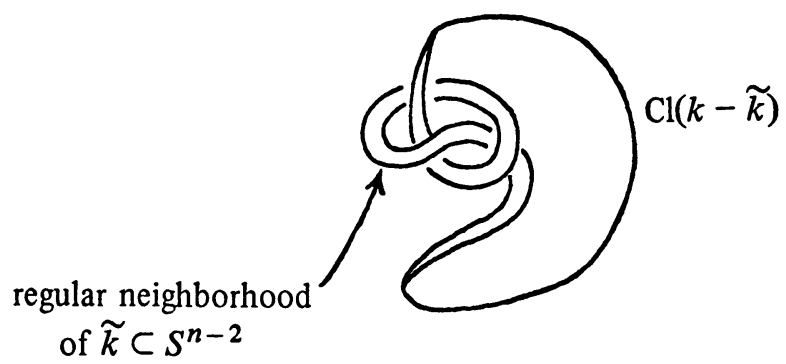

FIGURE 2

THEOREM 6.4. Let $k \subset S^{n-1}$ be a knotted submanifold and let $a \in \mathbf{Z}-\{0\}$. Then the a-twist spin $L_{a}(k)$ of $k$ is a fibered knotted submanifold of $S^{n}$; it is the binding of a book structure on $S^{n}$ with leaf $N_{-a}(\tilde{k})$.

Proof. The entire proof of the Interchange Theorem 1.10 may be carried over to our present context, excepting the construction of $\tilde{g}$, which no longer exists. In particular, the map $f: M \times S^{1} \rightarrow M \times S^{1}$ induces a map $\hat{f}$ : $N \times S^{1} \rightarrow S^{n}$ such that for all $\theta \in S^{1}, \hat{f}(\partial N \times \theta)=L_{1}(k)$. Thus, the 1-twist spin $L_{1}(k)$ of $k$ is a fibered knotted submanifold of $S^{n}$, which is the binding of a book with leaf $\hat{f}(N \times \theta) \simeq N$. By Lemma 2.3, and the property that the branch set $K^{n-2}$ is spun around $L_{1}(k)$ (see Definition 1.7), we may lift the book structure $\hat{f}(N \times \theta)$ to the total space of the branched covering $p_{a}$ : $S^{n} \rightarrow S^{n}$. Since each leaf $\hat{f}(N \times \theta)$ intersects $K^{n-2}$ in the disc $B_{\theta} \subset K^{n-2}$, and since $\hat{f}$ is orientation reversing, the leaf $p_{a}^{-1}(\hat{f}(N \times \theta))$ of the lifted book structure is $N_{-a}(\tilde{k})$.

ReMARK. Theorems 4.4 and 5.6 are true in the broader category of knotted submanifolds, since their proofs did not require $k$ to be a sphere.

7. Fox's conjecture is generalized, and we prove a result about iterated twist spinning. Recall (Definition 1.5) that two fibered knots $K, K^{\prime} \subset S^{n}$ are fiber equivalent if there is a homeomorphism $h:\left(S^{n}, K\right) \rightarrow\left(S^{n}, K^{\prime}\right)$ which preserves the fiber structure of their complements. In this section we prove:

TheOREM *. Let $k \subset S^{n-1}$ be a knot. Let $(a, b) \in \mathbf{Z} \times \mathbf{Z}-\{(0,0)\},(a, b)$ $=(g r, g s)$ where $g=$ g.c.d. $(a, b),(r, s) \in \mathbf{Z} \times \mathbf{Z}-\{(0,0)\}$ and g.c.d. $(r, s)=$ 1. Then the b-fold cyclic branched cover $M_{a, b}(k)$ of the a-twist spin $L_{a}(k) \subset S^{n}$ 
of $k \subset S^{n-1}$ is homeomorphic to $M_{0,8}(k)$ if either $r$ or $s$ is even, and to $M_{8,8}(k)$ if both $r$ and s are odd.

THEOREM **. Let $k \subset S^{n-1}$ be a knot. Let $(a, b) \in \mathbf{Z} \times \mathbf{Z}-\{(0,0)\}$, $(a, b)=(g r, g s)$ where $g=$ g.c.d. $(a, b), \quad(r, s) \in \mathbf{Z} \times \mathbf{Z}-\{(0,0)\}$ and g.c.d. $(r, s)=1$. Then the iterated twist spin $L_{a, b}(k) \subset S^{n+1}$ of $k \subset S^{n-1}$ is fiber equivalent to $L_{0, g}(k)$ if either $a$ or $b$ is even, and to $L_{g, g}(k)$ if both $a$ and $b$ are odd, where the fiber of the fibration of their complements over $S^{1}$ is given by Zeeman's theorem.

Since we cannot prove $M_{g, g}(k) \simeq M_{g, 0}(k)$, it is not clear to us that the knots $L_{g, g}(k)$ and $L_{g, 0}(k)\left(=L_{0, g}(k)\right)$ are fiber equivalent. Cameron Gordon claims that these knots are equivalent (not necessarily fiber equivalent) if $n>5$. He is also able to show that $M_{a, b}(k) \simeq M_{0, g}(k)$ if $n>5$ (the manifold $M_{a, b}(k)$ ball is the leaf of the book structure on $S^{n+1}$ with binding $\left.L_{a, b}(k)\right)$.

Definition 7.1. Let $Y^{n-2} \subset X^{n}$ be a codimension 2, properly embedded, oriented submanifold of the oriented manifold $X^{n}$, let $m \subset X-Y$ be a positive meridian for $Y$ in $X$ on which there is a distinguished point $p \in m$, and suppose $H_{1}(X-Y, \mathbf{Z}) \simeq \mathbf{Z}$ is generated by $[m]$. Then $\tau$ is a positive generator for the group $\mathbf{Z} / a \mathbf{Z}$ of covering translations of the $a$-fold cyclic branched covering $\pi: \tilde{X}_{a} \rightarrow X$ of $X^{n}$ branched along $Y^{n-2}, a \in \mathbf{Z}-\{0\}$, if there is a lift $\tilde{m} \subset \tilde{X}_{a}$ of $m$ such that

(i) $\partial \tilde{m}=\tau(\tilde{p})-\tilde{p}$ for some $\tilde{p} \in \pi^{-1}(p)$, if $a>0$,

(ii) $\partial \tilde{m}=\tilde{p}-\tau(\tilde{p})$ for some $\tilde{p} \in \pi^{-1}(p)$, if $a<0$.

Definition 7.2. Let $K \subset S^{n}$ be a knot, $a \in \mathbf{Z}-\{0\}$, and let $M_{a}(\tilde{K}) \rightarrow D^{n}$ be the $a$-fold cyclic branched cover of $D^{n}$ branched along the associated knotted disc $\tilde{K} \subset D^{n}$; let $\tau_{a}: M_{a}(\tilde{K}) \rightarrow M_{a}(\tilde{K})$ be a positive generator for the group of covering translations of $M_{a}(K)$. Define $T_{a}: M_{a}(\tilde{K}) \rightarrow M_{a}(\tilde{K})$ to be the following homeomorphism:

First note that $\partial M_{a}(\tilde{K})$ is a sphere $S^{n-1}$ on which the restriction $\tau_{a} \mid \partial M_{a}(K)$ of the covering translation $\tau_{a}$ is the rotation $r_{2 \pi / a}: S^{n-1} \rightarrow S^{n-1}$ with axis (fixed point set) the unknotted sphere $S^{n-3}=\partial \tilde{K} \subset \partial M_{a}(K)=S^{n-1}$. Let $\partial M_{a}(\tilde{K}) \times[0,1] \hookrightarrow M_{a}(\tilde{K})$ be a collar of the boundary of $M_{a}(\tilde{K})$ such that $\partial M_{a}(\tilde{K}) \times 0$ is identified with $\partial M_{a}(\tilde{K})$ (by projection to the first factor). We may assume that the restriction of $\tau_{a}$ to this entire collar is the rotation $r_{2 \pi / a} \times$ id: $S^{n-1} \times I \rightarrow S^{n-1} \times I$. Now define the homeomorphism $T_{a}$ to agree with $\tau_{a}$ on the complement of the collar, and inside the collar to slow to the identity on $\partial M_{a}(\tilde{K})$. On the collar, define $T_{a}$ by the equations:

$$
T_{a}(x, t)= \begin{cases}(x, t) & \text { if } x \in \partial M_{a}(\tilde{K}) \text { and } 0 \leqslant t \leqslant \frac{1}{3}, \\ \left(r_{(2 \pi / a)(3 t-1)}(x), t\right) & \text { if } x \in \partial M_{a}(\tilde{K}) \text { and } \frac{1}{3} \leqslant t<\frac{2}{3}, \\ \left(r_{2 \pi / a}(x), t\right) & \text { if } x \in \partial M_{a}(\tilde{K}) \text { and } \frac{2}{3}<t \leqslant 1 .\end{cases}
$$


Definition 7.3. Suppose $K \subset S^{n}$ is a fibered knot, $a \in \mathbf{Z}$ (we allow $a=0$ ). Let the associated book structure on $S^{n}$ with binding $K$ be given by $\left(S^{n}, F, h\right)$, where $\partial F=K$ and $h$ is a monodromy map for the book (see 1.1). Define a fiber-preserving homeomorphism $\tilde{T}_{a}: M_{a}(\tilde{K}) \rightarrow M_{a}(\tilde{K})$ as follows:

The $a$-fold cyclic branched covering $M_{a}(K) \rightarrow S^{n}$ of $S^{n}$ branched along $K$ is easily seen to have the book structure $\left(M_{a}(K), F, h^{a}\right)$, if $a \neq 0$; if $a=0$, this is the definition of $M_{a}(K)$ (see 5.3). Thus $M_{a}(K) \simeq F \times[0,1] /(x, 1) \sim$ $\left(h^{a}(x), 0\right)$ for all $x \in F,(x, 0) \sim(x, t)$ for all $x \in \partial F, t \in[0,1] ; M_{a}(K)$ has the orientation induced by the product orientation on $F \times[0,1]$. Define $\tilde{T}_{a}$ : $M_{a}(K) \rightarrow M_{a}(K)$ by $\tilde{T}_{a}(x, t)=\left(h^{-1}(x), t\right)$ for $(x, t) \in F \times[0,1]$, and let $\tilde{T}_{a}$ : $M_{a}(\tilde{K}) \rightarrow M_{a}(\tilde{K})$ be the restriction of $\tilde{T}_{a}: M_{a}(K) \rightarrow M_{a}(K)$ to $M_{a}(\tilde{K})=$ $M_{a}(K)$-ball.

LEMMA 7.4. Let $K \subset S^{n}$ be a knot, $a \in \mathbf{Z}-\{0\}$. Then the book structure on $S^{n+1}$ whose binding is the a-twist spin $L_{a}(K)$ of $K$ is given by $\left(S^{n+1}, M_{-a}(\tilde{K}), T_{-a}\right)$.

Proof. Let $\left(S^{n+1}, L_{1}(K)\right)$ be the pair $\left(D^{n} \times[0,1], \tilde{K} \times[0,1]\right) /(*)$ with identifications

$$
\begin{array}{ll}
(x, 0) \sim(x, t) & \text { for all } x \in \partial D^{n}, t \in[0,1], \\
(x, 1) \sim\left(T_{1}(x), 0\right) & \text { for all } x \in D^{n},
\end{array}
$$

and let $N=\partial D^{n} \subset D^{n} \times[0,1] /(*)$ be the standard unknotted $(n-1)=$ sphere in $S^{n+1}$. Let $\tilde{p}_{-a}: S^{n+1} \rightarrow S^{n+1}$ be the $(-a)$-fold cyclic branched covering of $S^{n+1}$ branched along the 1-twist spin $L_{1}(K)$.

We have the following commutative diagram:

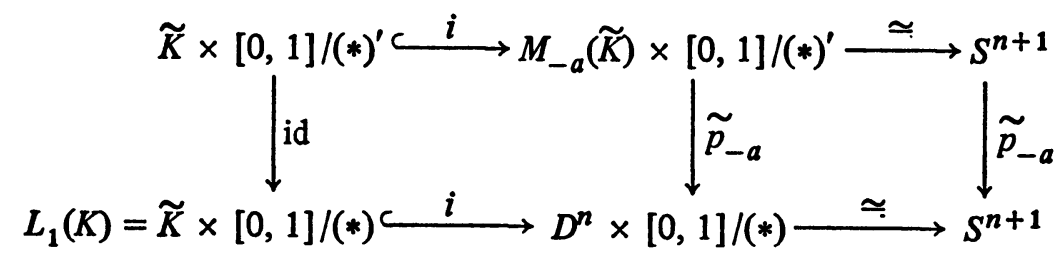

where the set of identifications (*)' is the lift of (*), and hence

$$
\begin{array}{ll}
(x, 0) \sim(x, t) & \text { for all } x \in \partial M_{-a}(\tilde{K}), t \in[0,1], \\
(x, 1) \sim\left(T_{-a}(x), 0\right) & \text { for all } x \in M_{-a}(\tilde{K}) .
\end{array}
$$

Now by Corollary 2.4, $L_{a}(K)$ is the inverse image $\tilde{p}_{-a}^{-1}(N)$ of the standard $(n-1)$-sphere $N \subset S^{n+1}$, under the branched covering $\tilde{p}_{-a}: S^{n+1} \rightarrow S^{n+1}$ of $S^{n+1}$ branched along $L_{1}(K)$. Hence

$L_{a}(K)=\tilde{p}_{-a}^{-1}(N)=\tilde{p}_{-a}^{-1}\left(\left[\partial D^{n}\right] \times[0,1] /(*)\right)=\left[\partial M_{-a}(\tilde{K})\right] \times[0,1] /(*)$.

The lemma is immediate. 
LEMMA 7.5. Let $K \subset S^{n}$ be a fibered knot, and let $\left(S^{n}, F, h\right)$ give a book structure on $S^{n}$ whose binding is $K=\partial F$. Let $a \in \mathbf{Z}-\{0\}$. Then $T_{a}: M_{a}(\tilde{K}) \rightarrow$ $M_{a}(\tilde{K})$ is isotopic, relative to $\partial M_{a}(\tilde{K})$, to the fiber preserving homeomorphism $\tilde{T}_{a}: M_{a}(\tilde{K}) \rightarrow M_{a}(\tilde{K})$. Hence, the book structure $\left(S^{n+1}, M_{-a}(\tilde{K}), T_{-a}\right)$ on $S^{n+1}$ whose binding is the a-twist spin $L_{a}(K)$ of $K$ is also given by $\left(S^{n+1}, M_{-a}(\tilde{K}), \tilde{T}_{-a}\right)$.

Proof. First, $T_{1}:\left(D^{n}, \tilde{K}\right) \rightarrow\left(D^{n}, \tilde{K}\right)$ is isotopic to $\tilde{T}_{1}:\left(D^{n}, \tilde{K}\right) \rightarrow\left(D^{n}, \tilde{K}\right)$ relative to $\partial D^{n}$ and $\tilde{K}$, because $K \subset S^{n}$ is a fibered knot. Denote the isotopy by $T_{1, t}:\left(D^{n}, \tilde{K}\right) \rightarrow\left(D^{n}, \tilde{K}\right)$, where $T_{1,0}=T_{1}$ and $T_{1,1}=\tilde{T}_{1}$, and let $\pi$ : $\left(M_{a}(\tilde{K}), \tilde{K}\right) \rightarrow\left(D^{n}, \tilde{K}\right)$ be the $a$-fold cyclic branched covering of $D^{n}$ branched along $\tilde{K}$. Then, as shown in the following commutative diagram, the isotopy $T_{1, t}$ lifts to an isotopy $T_{a, t}:\left(M_{a}(\tilde{K}), \tilde{K}\right) \rightarrow\left(M_{a}(\tilde{K}), \tilde{K}\right)$ relative to $\partial M_{a}(\tilde{K})$ and $\tilde{K}$, between $T_{a, 0}=T_{a}$ and $T_{a, 1}=\tilde{T}_{a}$ :

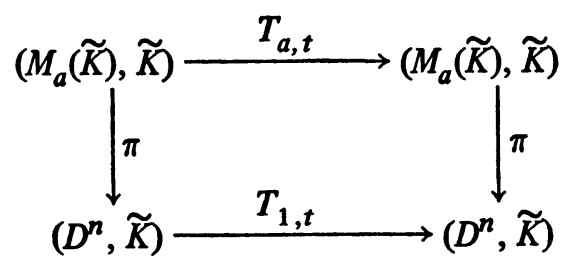

This automatically implies the second part of the lemma.

Recall that if $k \subset S^{n-1}$ is a knot, $a, b \in \mathbf{Z}$ and $b \neq 0$, then $M_{a, b}(k) \rightarrow S^{n}$ denotes the $b$-fold cyclic branched covering of $S^{n}$, branched along the $a$-twist spin $L_{a}(k) \subset S^{n}$ of $k \subset S^{n-1}$. If $b=0$, the definition of $M_{a, b}(k)$ is given by 5.4. There is a natural identification of $L_{a}(k) \subset S^{n}$ with its inverse image in $M_{a, b}(k)$ given by the covering projection, and this is what we will mean by the notation $L_{a}(k) \subset M_{a, b}(k)$.

THEOREM 7.6. Let $k \subset S^{n-1}$ be a knot, $a, b \in \mathbf{Z}-\{0\}$. Then there is $a$ homeomorphism $H:\left(M_{a, b}(k), L_{a}(k)\right) \rightarrow\left(M_{a, b \pm 2 a}(k), L_{a}(k)\right)$.

Proof. It is an easily proven property of the book structure $(M, F, h)$ on a manifold $M$, that the $r$-fold cyclic branched covering $\pi: M_{r} \rightarrow M$ of $M$ branched along the binding $\partial F$ of the book, $r \in \mathbf{Z}-\{0\}$, has a book structure given by $\left(M_{r}, F, h^{r}\right)$.

Consequently, if $r \in \mathbf{Z}$, Lemma 7.4 and Definition 5.4 imply that the manifold $M_{a, r}(k)$ has a book structure with leaf $M_{a}(\tilde{k})$ and monodromy $\left(T_{-a}\right)^{r}: M_{-a}(\tilde{k}) \rightarrow M_{-a}(\tilde{k})$. Next, we observe that the homeomorphisms $\left(T_{-a}\right)^{b}$ and $\left(T_{-a}\right)^{b \pm 2 a}$ agree outside of the collar $\left[\partial M_{-a}(\tilde{k})\right] \times\left[\frac{1}{3}, \frac{2}{3}\right]$. Further, their difference

$$
\left(T_{-a}\right)^{ \pm 2 a}:\left[\partial M_{-a}(\tilde{k})\right] \times\left[\frac{1}{3}, \frac{2}{3}\right] \rightarrow\left[\partial M_{-a}(\tilde{k})\right] \times\left[\frac{1}{3}, \frac{2}{3}\right]
$$


on this collar is isotopic to the identity, relative to the boundary $\left[\partial M_{-a}(\tilde{k})\right] \times\left\{\frac{1}{3}, \frac{2}{3}\right\}:$

Since $\partial M_{-a}(\tilde{k}) \simeq S^{n-1}$ and the monodromy $T_{-a}$ is defined on the collar $S^{n-1} \times\left[\frac{1}{3}, \frac{2}{3}\right]$ by $T_{-a}(x, t)=\left(r_{2 \pi /-a(3 t-1)}(x), t\right)$, we have that $\left(T_{-a}\right)^{ \pm 2 a}$ : $S^{n-1} \times\left[\frac{1}{3}, \frac{2}{3}\right] \rightarrow S^{n-1} \times\left[\frac{1}{3}, \frac{2}{3}\right]$ defined by $\left(T_{-a}\right) \pm 2 a(x, t)=$ $\left(r_{ \pm 4 \pi(3 t-1)}(x), t\right)$ represents the trivial element of $\pi_{1}(\mathrm{SO}(n))$.

Now we apply another property of book structures, that $(M, F, h)$ is equivalent to $\left(M, F, h^{\prime}\right)$ if $h$ and $h^{\prime}$ are isotopic relative to $\partial F$.

REMARK. In fact, it is clear from the proof that

(a) $H$ carries a tubular neighborhood $N$ of $L_{a}(k) \subset M_{a, b}(k)$ to a tubular neighborhood $N^{\prime}$ of $L_{a}(k) \subset M_{a, b \pm 2 a}(k)$.

(b) the spaces $M_{a, b}(k)-N$ and $M_{a, b \pm 2 a}(k)-N^{\prime}$ are fiber bundles over $S^{1}$ with the same fiber, and the same monodromy.

(c) $H: M_{a, b}(k)-N \rightarrow M_{a, b \pm 2 a}(k)-N^{\prime}$ is the identity.

THEOREM 7.7. Let $k \subset S^{n-1}$ be a knot, $a, b \in \mathbf{Z}-\{0\}$. Then the iterated twist spun knots $L_{a, b}(k), L_{a, b \pm 2 a}(k) \subset S^{n+1}$ are fiber equivalent, fibered knots.

Proof. Let $\hat{H}: M_{-b}\left(\widetilde{\left.L_{a}(k)\right)} \rightarrow M_{-b \pm 2 a} \widetilde{\left(L_{a}(k)\right)}\right.$ be the restriction $H \mid$ : $M_{a,-b}(k)$-ball $\rightarrow M_{a,-b \pm 2 a}$-ball of $H$, to the manifold $M_{a,-b}(k)$ with an open $n$-ball centered at a point of $L_{a}(k) \subset M_{a-b}(k)$ removed from the interior of the tubular neighborhood $N$ of $L_{a}(k)$. Now by Lemma 7.5 and Corollary 5.6, the knot $L_{a, r}(k) \subset S^{n+1}, r \in \mathbf{Z}$, is the binding of a book structure on $S^{n+1}$ with leaf $M_{-r}\left(\widetilde{\left.L_{a}(k)\right)}=M_{a,-r}(k)\right.$-ball, and monodromy $\tilde{T}_{-r}: M_{-r}\left(\widetilde{L_{a}(k)}\right) \rightarrow$ $M_{-r}\left(\widetilde{\left.L_{a}(k)\right)}\right.$. It may be assumed that $\tilde{T}_{-r}: M_{-r}\left(\widetilde{\left.L_{a}(k)\right)} \rightarrow M_{-r}\left(\widetilde{\left.L_{a}(k)\right)}\right.\right.$ is the identity on a tubular neighborhood of $\widetilde{L_{a}(k)}$ which includes the collar $\left[\partial M_{-a}(k)\right] \times\left[\frac{1}{3}, \frac{2}{3}\right] \times S^{1}$. Since $\hat{H}$ is the identity off of this collar (see part (c) of the Remark), it follows that $\tilde{T}_{-b}=\hat{H}^{-1} \circ \tilde{T}_{-b \pm 2 a} \circ \hat{H}: M_{-b}\left(L_{a}(k)\right) \rightarrow$ $M_{-b}\left(L_{a}(k)\right)$. Thus $\hat{H}$ may be extended to a fiber-preserving homeomorphism

$$
\left(S^{n+1}, M_{-b}\left(\widetilde{L_{a}(k)}\right), \tilde{T}_{-b}\right) \rightarrow\left(S^{n+1}, M_{-b \pm 2 a}\left(\widetilde{L_{a}(k)}\right), \tilde{T}_{-b \pm 2 a}\right)
$$

of these book structures on $S^{n+1}$, giving the desired fiber equivalence $\left(S^{n+1}, L_{a, b}(k)\right) \rightarrow\left(S^{n+1}, L_{a, b \pm 2 a}(k)\right)$.

\section{COROLlaRY 7.8. Theorems * and **.}

Proof. We have objects, either $M_{a, b}(k)$ or $L_{a, b}(k)$, indexed by pairs of integers $(a, b) \in \mathbf{Z} \times \mathbf{Z}-\{(0,0)\}$. Let us denote the object simply by the pair $(a, b)$. We also have an equivalence relation between objects, namely homeomorphism for the manifolds $M_{a, b}(k)$ and fiber equivalence for the fibered knots $L_{a, b}(k)$; we will denote the equivalence by $(a, b) \sim\left(a^{\prime}, b^{\prime}\right)$.

Observe that we have proved these relations:

(1) $(a, b) \sim(a,-b)$, 
(2) $(a, b) \sim(b, a)$.

(3) $(a, b) \sim(a, b+2 a)$.

As a consequence of (a), (b), (c) we have:

(4) $(a, b) \sim(a, b-2 a)$.

By (1) and (2) we need only consider the pairs $(a, b)$ with $b>a>0$. Suppose $b \neq a$ and $a \neq 0$. If $b>2 a$ then $(a, b) \sim(a, b-2 a)$. If $b<2 a$ then $(a, b) \sim(a, 2 a-b)$. In either case we have $(a, b) \sim\left(a^{\prime}, b^{\prime}\right)$ where $b^{\prime}>a^{\prime}>0$ and $a^{\prime}<a, b^{\prime}<b$.

Repeating this procedure we finally arrive at $(a, b) \sim\left(a^{\prime}, b^{\prime}\right)$; where either $a^{\prime}=b^{\prime}=g$ or $a^{\prime}=0, b^{\prime}=g$ and $g \in \mathbf{Z}^{+}$.

We now observe the following facts:

(a) If $(a, b) \sim\left(a^{\prime}, b^{\prime}\right)$, then g.c.d. $(a, b)=$ g.c.d. $\left(a^{\prime}, b^{\prime}\right)$.

(b) If $(a, b) \sim\left(a^{\prime}, b^{\prime}\right)$ and $(a, b)=(g r, g s),\left(a^{\prime}, b^{\prime}\right)=\left(g r^{\prime}, g s^{\prime}\right)$ as in the statement of Theorems * and **, then $r$ and $s$ are both odd $\Leftrightarrow r^{\prime}$ and $s^{\prime}$ are both odd.

It follows that if $(a, b) \sim(0, g)$ or $(a, b) \sim(g, g)$, then $g=$ g.c.d. $(a, b)$. Also if $(a, b)=(g r, g s)$, then $(a, b) \sim(0, g)$ precisely when either $r$ or $s$ is even, and $(a, b) \sim(g, g)$ when both $r$ and $s$ are odd.

8. Spinning symmetric arc collections. We believe that the exposition of this section will be clearest if we restrict ourselves to dimension 3. It is understood, however, that dimensions 3 and 4 may be replaced by $n-1$ and $n$, respectively, in the statement of the definitions, Theorem 8.5 and Corollary 8.6, and that these two results will still be correct.

For a discussion of 2-symmetric arc collections, the reader is referred to Dennis Roseman's article [5].

Definition 8.1. A collection $C$ of $a$ embedded arcs in $D^{3}$ with disjoint interiors, whose common boundary is the poles $K^{0} \subset \partial D^{3}$, is a-symmetric if it is invariant under the rotation $r_{2 \pi / a}: D^{3} \rightarrow D^{3}$.

Definition 8.2. The quotient arc $k(C) \subset D^{3}$ of $C$ is the image of $C$ under the projection to the space of orbits of the action $r_{2 \pi / a}: D^{3} \rightarrow D^{3}$.

Definition 8.3. Let $C$ be an $a$-symmetric collection of arcs in $D^{3}$. The knot associated with $C$ is the union of the quotient $\operatorname{arc} k(C)$ and the unknotted arc which is the fixed point set or axis, of the rotation $r_{2 \pi / a}: D^{3} \rightarrow D^{3}$. (See Figure 3.)

Definition 8.4. Let $C$ be an $a$-symmetric collection of arcs in $D^{3}$. A knot $L \subset S^{4}$ is called the spin of $C$ about the unknotted sphere $K^{2} \subset S^{4}$ if

$$
L \cap D_{\theta}^{3}=i_{\theta} r_{\theta / a}(C) \text {. }
$$

We denote the spin of $C$ about $K$ by $L(C)$.

REMARK. The knot $L(C)$ is locally flat at each pole, since the intersection 
(i)

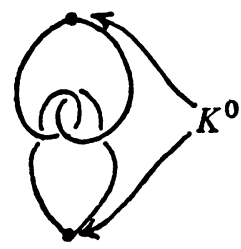

2-symmetric $C$

(ii)

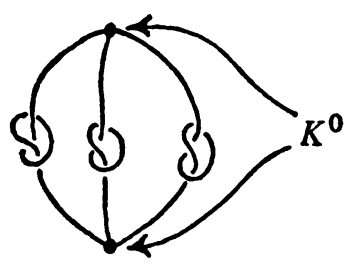

3-symmetric $C$

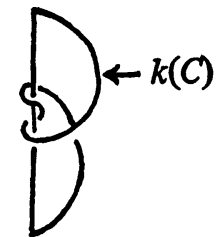

the associated knot

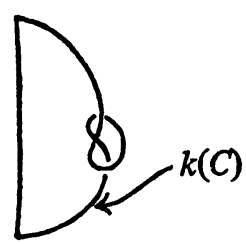

the associated knot

FIGURE 3

of a regular neighborhood of the pole with $L(C)$ is an $(a, 1)$-torus knot (which is trivial).

Notation. If $C$ is an $a$-symmetric collection of arcs in $D^{3}$ with quotient arc $k(C)$, let $L$ denote the spin of the 1-symmetric collection $k(C)$ in $S^{4}$. Let $\tilde{K}$ denote the spin in $S^{4}$ of the unknotted arc which is the fixed point set, or axis, of the rotation $r_{\theta / a}: D^{3} \rightarrow D^{3}$. There is an obvious deformation of $\tilde{K}$ to the standard unknotted 2-sphere $K$.

Let $p_{a}: D^{3} \rightarrow D^{3}$ be the $a$-fold cyclic branched covering of $D^{3}$ branched along the unknotted axis of the rotation $r_{\theta / a}: D^{3} \rightarrow D^{3}$. (This is just the restriction to the unit ball $D^{3} \subset S^{3}$, of the map $p_{a}: S^{3} \rightarrow S^{3}$ defined in \$2.) Note that $p_{a}(C)=k(C)$.

Let the $a$-fold cyclic branched covering $P_{a}: S^{4} \rightarrow S^{4}$ of $S^{4}$ branched along $\tilde{K}$ be induced by

$$
p_{a} \times \text { id: } D^{3} \times S^{1} \rightarrow D^{3} \times S^{1} .
$$

Note that $P_{a}(L(C))=L$.

THEOREM 8.5. Let $C$ be an a-symmetric collection of arcs in $D^{3}$, and let $k \subset S^{3}$ be the knot associated with $C$. Then $L(C) \cong L_{a}(k)$ : the spin of the collection $C$ about the unknotted sphere $K^{2} \subset S^{4}$ is ambient isotopic to the $a$-twist spin of the knot $k$ associated to $C$.

Proof. We will use the characterization of $L(C)$ given above; namely, $L(C)$ is the inverse image $P_{a}^{-1}(L)$ of $L$ under the branched covering map $P_{a}$ : 
$S^{4} \rightarrow S^{4}$. Then by Lemma 2.3 , the theorem will be proved if we can exhibit a homeomorphism $h: S^{4} \rightarrow S^{4}$ such that $h(\tilde{K})=K$ and $h(L)=L_{1}(k)$.

Let $h: S^{4} \rightarrow S^{4}$ be the homeomorphism constructed in Theorem 1.10 which interchanges $K$ and $\tilde{K}$. Then $h(L)=L_{1}(k)$ :

As in the proof of 1.10 the 4-tuple $\left(S^{4}, \tilde{K}, K, L\right)$ is homeomorphic to the 4-tuple $\left(M \times S^{1}, A \times S^{1}, B \times S^{1}, k(C) \times S^{1}\right)$ after the identifications (i) and (ii). Therefore it is equivalent to check that $\tilde{h}\left(k(C) \times S^{1}\right)=\tilde{k} \times S^{1}$, or, for each $\theta \in S^{1}, \tilde{h}\left(k(C) \times S^{1}\right) \cap(M \times \theta)=\tilde{k} \times \theta$, where $\tilde{k} \subset D^{3}$ is the knotted arc associated to $k \subset S^{3}$. The reader should review the definitions of the maps $f, g, \tilde{h}: M \times S^{1} \rightarrow M \times S^{1}$ and $\tilde{g}: M \rightarrow M$ in the proof of 1.10 before beginning what follows:

$$
\begin{aligned}
\tilde{h}\left(k(C) \times S^{1}\right) \cap(M \times \theta) & =\tilde{h}\left[\left(k(C) \times S^{1}\right) \cap(\tilde{h})^{-1}(M \times \theta)\right] \\
& =\tilde{h}\left[\left(k(C) \times S^{1}\right) \cap(\tilde{h})^{-1}\left(\bigcup_{\phi \in S^{1}} F_{\phi} \times \theta\right)\right] \\
& =\tilde{h}\left[\left(k(C) \times S^{1}\right) \cap\left(\bigcup_{\phi \in S^{\prime}} F_{\phi} \times(-\theta-\phi)\right)\right] \\
& =\tilde{h}\left[\bigcup_{\phi \in S^{1}}\left(k(C) \times S^{1}\right) \cap\left(F_{\phi} \times(-\theta-\phi)\right)\right] \\
& =\tilde{h}\left[\bigcup_{\phi \in S^{1}}\left(k(C) \cap F_{\phi}\right) \times(-\theta-\phi)\right] \\
& =g \circ f\left[\bigcup_{\phi \in S^{1}}\left(k(C) \cap F_{\phi}\right) \times(-\theta-\phi)\right] \\
& =g\left[\bigcup_{\phi \in S^{1}}\left(k(C) \cap F_{\phi}\right) \times \theta\right] \\
& =g[k(C) \times \theta]=\tilde{g}[k(C)] \times \theta=\tilde{k} \times \theta .
\end{aligned}
$$

(1)

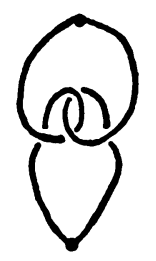

2-symmetric $C$
(2)

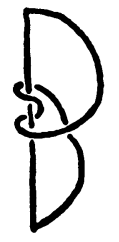

the associated knot

Figure 4 
(3)

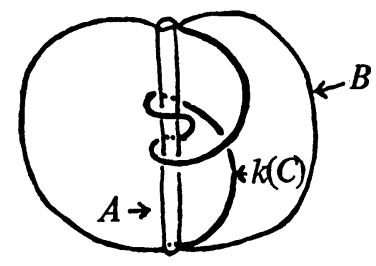

(4)

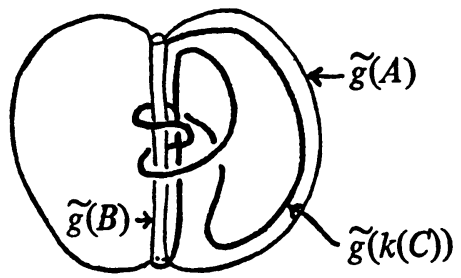

(5)

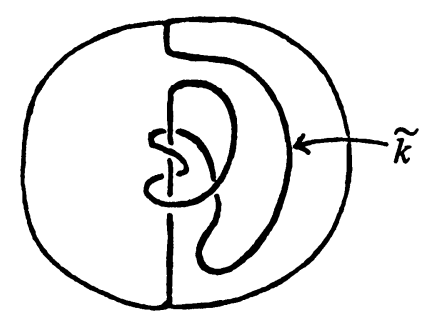

the knotted arc $\widetilde{k}$ corresponding to the knot $k$

\section{FIGURE 4 (continued)}

The final step, which asserts the equality $\tilde{g}[k(C)]=\tilde{k}$ of $\tilde{g}[k(C)]$ and the knotted arc $\tilde{k}$ corresponding to the knot $k$ associated with the arc collection, is left to the reader, with the aid of Figure 4.

\section{REFERENCES}

1. A. Durfee and L. Kauffman, Periodicity of branched cyclic covers, Math. Ann. 218 (1975), 157-174.

2. R. H. Fox, Covering spaces with singularities, Algebraic geometry and topology-A symposium in honor of S. Lefschetz, Princeton Univ. Press, Princeton, N. J., 1957, pp. 244-257.

3. Some n-dimensional manifolds that have the same fundamental group, Michigan Math. J. 15 (1968), 187-189.

4. D. L. Goldsmith, Symmetric fibered links, knots, groups and 3-manifolds, papers dedicated to the memory of R. H. Fox, Ann. of Math. Studies, Princeton Univ. Press, Princeton, N. J., 1975, pp. 3-25.

5. D. Roseman, Half-twist spinning of arc pairs and inversions of knots, Illinois J. Math. (to appear).

6. E. C. Zeeman, Twisting spun knots, Trans. Amer. Math. Soc. 115 (1965), 471-495.

Department of Mathematics, University of Michigan, ANn Arbor, Michigan 48109

Department of Mathematics, University of Illinois at Chicago Circle, Chicago, ILLINOIS 60680 